\title{
Diversidad de Carabidae (Insecta: Coleoptera) en distintos ambientes de un agroecosistema del sudeste bonaerense, Argentina
}

\author{
Adela V. Castro ${ }^{\bowtie}$; Darío P. Porrini \& Armando C. Cicchino \\ GENEBSO, INBIOTEC, CONICET, FCEyN, UNMdP, Mar del Plata, Argentina.
}

\begin{abstract}
Resumen. Los coleópteros de la familia Carabidae conforman gran parte de la biodiversidad de los suelos de los agroecosistemas y por eso son buenos organismos modelo para estudiar la composición y la estructura de las comunidades. Los objetivos de este trabajo fueron: a) analizar la diversidad de Carabidae (riqueza, actividad, composición y estructura del ensamble) en ambientes con distinto uso antrópico; b) conocer la asociación de las especies de Carabidae con la vegetación herbácea; c) analizar la proporción de especies de acuerdo a la cantidad de ambientes que ocupan, su capacidad de dispersión por medio del vuelo y su sinantropía; d) identificar potenciales especies típicas de los distintos ambientes, y e) registrar la presencia de larvas. El estudio se realizó en un agroecosistema de la Laguna Nahuel Rucá $\left(37^{\circ} 37^{\prime} 04^{\prime \prime}\right.$ S - $\left.57^{\circ} 25^{\prime} 16^{\prime \prime} \mathrm{O}\right)$. Se seleccionaron distintos ambientes de muestreo: tres ambientes de bosque, tres ambientes abiertos y un ambiente mixto, que fueron muestreados mensualmente mediante trampas "pitfall" a lo largo de un año. Se elaboró un índice para clasificar el grado de modificación antrópica de cada ambiente. Los valores más altos de riqueza se observaron en los ambientes de bosque. Los ensambles de Carabidae se diferenciaron en función de la estructura del hábitat. Se identificaron especies típicas de los ambientes abiertos y de los ambientes de bosque. La proporción de especies macrópteras y sinantrópicas tendió a ser mayor en los ambientes abiertos, mientras que no se encontraron diferencias en la proporción de eurítopas. Diez especies de carábidos se asociaron con las familias Convolvulaceae, Apiaceae y Fabaceae en primavera. Siete especies de larvas se registraron principalmente en ambientes de bosque durante el verano. Se puede concluir que el uso del suelo y la estructura del ambiente influyen sobre la diversidad de carábidos en la escala local.
\end{abstract}

[Palabras clave: carábidos, talar, biodiversidad, entomología, sistemática, Buenos Aires]

\begin{abstract}
Diversity of Carabidae (Insecta: Coleoptera) in different environments in an agroecosystem from southeast Buenos Aires, Argentina. The coleopterans of the family Carabidae conform a large part of the soil biodiversity from agroecosystems, hence they are good model organism to study the composition and structure of communities. The aims of this contribution were to: a) analyze the diversity of Carabidae (species richness, activity, composition and structure of assemblages) in environments with different anthropic use; b) know the association of Carabidae species with herbaceous vegetation; c) analyze the proportion of species according to their specialisation to habitat requirements, capacity of dispersion in relation to wing dimorphism and sinanthropism, and d) identify typical species of different environments and e) record the presence of larvae. The study was conducted in an agroecosystem from Laguna Nahuel Ruca (37 $\left.37^{\prime} 04^{\prime \prime} \mathrm{S}-57^{\circ} 25^{\prime} 16^{\prime \prime} \mathrm{W}\right)$. We selected different sampling environments: three forest environments, three open environments and a mixed environment; all of them were sampled monthly with "pitfall" traps during one year. We made a ranking to measure the degree of human modification of each environment. Higher values of species richness were recorded in forest environments. Carabidae assemblages differed according to habitat structure. We identified typical species of open environments as well as forest environments. The proportion of macropterous and synanthropic species tended to be higher in open environments, while there was no difference in the proportion of eurytopic species. Ten species of Carabidae were associated with families Convolvulaceae, Apiaceae and Fabaceae in spring. Seven species of larvae were recorded mainly in forest environments during summer. We concluded that land use and the structure of environment influence the local biodiversity of Carabidae.
\end{abstract}

[Keywords: ground beetles, tala forest, biodiversity, entomology, systematic, Buenos Aires]

Editor asociado: David Bilenca

adelamdp@gmail.com
Recibido: 29 de diciembre de 2015

Aceptado: 12 de junio de 2017 


\section{INTRODUCCIÓN}

Las prácticas agrícolo-ganaderas actuales implican una simplificación del paisaje, ya que reemplazan la diversidad natural por unas pocas especies de plantas cultivadas y animales domésticos (Altieri 1999). La actividad productiva afecta a casi todos los procesos ecológicos, desde el comportamiento delos individuos y las dinámicas poblacionales hasta la composición y estructura de las comunidades y los flujos de materia y energía (Bilenca et al. 2012). En los últimos años, la actividad agropecuaria se intensificó en la región pampeana, en particular en la cuenca de Mar Chiquita. El avance agrícola ocurrió en mayor grado en suelos elevados, como en los albardones de lagunas y arroyos. Sin embargo, la conservación de los suelos que aún existen cubiertos por vegetación nativa, como pastizales y talares, puede estar en riesgo si continúa la expansión de la ganadería y el reemplazo de la vegetación natural por pasturas cultivadas (Maceira et al. 2005).

Los agroecosistemas deben ser considerados como un patrón complejo de parches de hábitats cultivados y no cultivados (Marasas et al. 2010). En ellos, la biodiversidad interviene en procesos que favorecen su sustentabilidad y que van más allá de la producción de alimentos, combustibles e ingresos. Por ejemplo, la macrofauna edáfica participa de forma activa en el ciclo de nutrientes a través de la fragmentación de residuos vegetales, de la estimulación de la actividad microbiana y de la regulación de los organismos indeseables, entre otros procesos. Todo ello conduce a un rendimiento sustentable, a la conservación de la energía y a una menor dependencia de insumos externos (Altieri 1999). A partir de esto, para entender cuál es la contribución de los invertebrados a la calidad del suelo (Stork and Eggleton 1992) surge la necesidad de conocer la estructura de las comunidades edáficas y cómo las afectan las distintas prácticas de manejo. Un primer acercamiento lo ofrecen los inventarios de especies, que permiten conocer el estado de la biodiversidad para prevenir o remediar el impacto antrópico en distintos ambientes (Wink et al. 2005). Esto permite repensar el diseño de los agroecosistemas en función de lograr modos de producción cada vez más independientes de insumos químicos, revalorizar el papel de los controladores biológicos de plagas y facilitar a los productores la transición agroecológica (Marasas 2012).
Entrelosintegrantes dela macrofauna edáfica, los carábidos son buenos modelos de estudio, ya que la mayoría de sus especies, tanto en estadio adulto como larval, son depredadoras de plagas agrícolas, son sensibles a prácticas de manejo como el pastoreo, la fertilización, etc. (Rainio and Niemelä 2003; Cicchino et al. 2011; Castro et al. 2014) y presentan gran abundancia, diversidad y funcionalidad (Koivula 2011)

Por estos motivos, el objetivo general de este trabajo fue realizar un monitoreo de la diversidad de coleópteros de la familia Carabidae presentes en ambientes con distinto uso dentro del agroecosistema del talar de Laguna Nahuel Rucá. A su vez, se pretendió investigar algunos aspectos ecológicos de la carabidofauna local e interpretar la potencialidad de estos organismos como bioindicadores del estado de conservación de ambientes sujetos a prácticas antrópicas diversas. Los objetivos específicos que se plantearon fueron: a) analizar la diversidad de Carabidae (riqueza específica, actividad, composición y estructura del ensamble); b) describir estacionalmente la asociación de las especies más activas de carábidos con la vegetación herbácea; c) analizar la proporción de especies eurítopas, macrópteras y sinantrópicas; d) identificar potenciales especies o grupos de especies indicadoras de los distintos ambientes analizados, y e) registrar la presencia de larvas en los distintos ambientes.

Nuestra hipótesis postula que la riqueza de especies es menor en ambientes altamente disturbados, que la composición y estructura del ensamble difieren según el uso del hábitat y que se pueden reconocer especies indicadoras del estado del ambiente. De acuerdo con la bibliografía existente, es esperable que en ambientes muy disturbados se vea favorecida la abundancia de especies eurítopas (Niemelä 2001), sinantrópicas o hemisinantrópicas (Cicchino 2003), así como de las especies con alta vagilidad (i.e., macrópteras con capacidad de vuelo activo), que suelen ser buenas colonizadoras, capaces de mantener poblaciones estables en parches pequeños y aislados (Niemelä 2001).

\section{Materiales y Métodos}

\section{Área de estudio}

El estudio se realizó en la Estancia Nahuel Rucá, una propiedad privada ubicada al 
margen de la Laguna Nahuel Rucá (3737'04" S - 57 $25^{\prime} 16^{\prime \prime} \mathrm{O}$ ), incluida en la zona buffer de la Reserva Provincial y de Biósfera Mar Chiquita. Presenta características únicas dentro de la región, ya que conserva en muy buen estado un bosque nativo de talas (Celtis ehrenbergiana (Klotzsch) Liebm., Celtidaceae) que no se repite con estas características en ningún otro sitio del sudeste bonaerense. El sistema de estudio abarcó una superficie aproximada de 15 ha en la que se seleccionaron siete ambientes de muestreo distintos (Figura 1). Por sus características fitosociológicas, éstos fueron discriminados de la siguiente forma: a tres se los denominó "ambientes de bosque": el talar, el borde del talar y un borde de talas y cipreses (Cupressus macrocarpa Hartw.) bordeando un potrero ("borde potrero"); a otros tres se los llamó "ambientes abiertos": dos cultivos forrajeros de maíz (CI y CII) de pequeña escala y un potrero de pastoreo. Por último, se seleccionó un "ambiente mixto", el jardín parquizado con especies nativas y ornamentales exóticas, conformando un mosaico de zonas arboladas y abiertas. En CII, antes de la siembra, el propietario realizó aplicaciones de glifosato a las dosis recomendadas para controlar las hierbas. En octubre de 2010, se sembraron semillas (NIDERA®) de maíz híbrido (Zea mays) en ambos cultivos por siembra directa. En CII, el terreno fue tratado con las dosis recomendadas del insecticida Karate ${ }^{\circledR}$ (60-75 $\mathrm{cm}^{3} / \mathrm{ha}$ ), cuyo principio activo es el piretroide lambda cihalotrina, para el control de la isoca de la espiga en maíz Helicoverpa zea (Boodie, 1850). En CI, la dosis de herbicida aplicada fue aproximadamente la mitad de la aplicada en
CII, $700 \mathrm{~cm}^{3} /$ ha, y no se aplicó insecticida. En los meses de julio y agosto se ingresó el ganado a los cultivos para forrajear, y en octubre de 2011 se volvió a realizar la siembra directa de maíz, con aplicaciones convencionales de agroquímicos en ambos cultivos.

Debido a que todos los ambientes tienen un uso antrópico diverso, para caracterizar el grado de modificación en cada ambiente por el ser humano se elaboró un índice que tuvo en cuenta distintos aspectos: a) porcentaje de cobertura por especies arbóreas exóticas, b) porcentaje de suelo desnudo, c) porcentaje de área ocupada con edificaciones, d) presencia de cultivo forrajero, e) aplicación de agroquímicos, f) pastoreo por ganado, y g) corte periódico de césped. A cada ítem se le asignó un valor (Tabla 1) y se sumó el total para cada ambiente. Los valores más altos del índice indican mayor grado de modificación del ambiente.

\section{Muestreo de carábidos}

Se realizó mediante trampas de caída o trampas conocidas como "pitfall" (Adis 2002). En cada ambiente se colocaron seis trampas pitfall separadas entre sí por una distancia mínima de $10 \mathrm{~m}$, dispuestas en una transecta lineal. Estas consistieron en envases plásticos de $11 \mathrm{~cm}$ de diámetro y $12 \mathrm{~cm}$ de profundidad, con una capacidad de $850 \mathrm{~mL}$, y fueron enterradas con su boca $1-2 \mathrm{~cm}$ por debajo de la superficie del suelo. Las mismas contenían aproximadamente $400 \mathrm{~mL}$ de una solución compuesta por $200 \mathrm{~g}$ de sulfito de sodio, $200 \mathrm{~mL}$ de formol y $25 \mathrm{~mL}$ de detergente

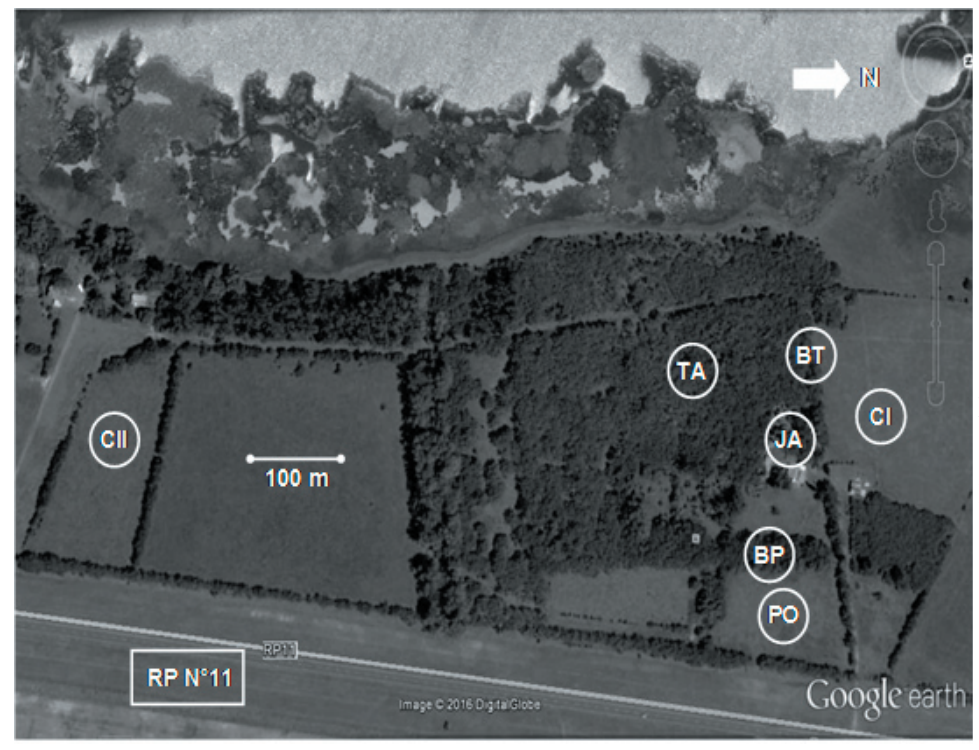

Figura 1. Área de estudio. BP: borde potrero; BT: borde talar; CI: cultivo I; CII: cultivo II; JA: jardín parquizado; TA: talar centenario; PO: potrero; RP $\mathrm{N}^{\circ} 11$ : Ruta Provincial No 11.

Figure 1. Study area. BT: tala forest edge; BP: pasture edge; CI: crop with low doses of pesticides; CII: crop with conventional doses of pesticides; JA: garden; PO: grazing pasture area; TA: tala forest; RP $\mathrm{N}^{\circ} 11$ : Provincial Route $\mathrm{N}^{\mathrm{o}} 11$. 
Tabla 1. Evaluación del grado de disturbio de los distintos ambientes.

Table 1. Assessment of the degree of disturbance of the different environments.

\begin{tabular}{|c|c|c|c|c|c|c|c|c|}
\hline Características & Valor & BT & $\mathrm{BP}$ & $\mathrm{CI}$ & $\mathrm{CII}$ & JA & $\mathrm{PO}$ & TA \\
\hline Árboles exóticos & $\begin{array}{c}0=0-25 \% ; 1=25-50 \% \\
2=50-75 \% ; 3=75-100 \%\end{array}$ & 0 & 2 & 0 & 0 & 2 & 0 & 0 \\
\hline Suelo desnudo & $\begin{array}{c}0=0-25 \% ; 1=25-50 \% \\
2=50-75 \% ; 3=75-100 \%\end{array}$ & 0 & 0 & 0 & 0 & 1 & 0 & 0 \\
\hline Edificaciones & $\begin{array}{l}0=0-12 \% ; 1=12-25 \% \\
2=25-37 \%, 4=37-50 \% ; \\
5=50-62 \% ; 6=62-75 \% ; \\
7=75-87 \%, 8=87-100 \%\end{array}$ & 0 & 0 & 0 & 0 & 1 & 0 & 0 \\
\hline Cultivo & $0=$ no; $1=$ sí & 0 & 0 & 1 & 1 & 0 & 0 & 0 \\
\hline Aplicación de agroquímicos & $0=$ ausente $; 1=$ reducida $; 2=$ convencional & 0 & 0 & 1 & 2 & 0 & 0 & 0 \\
\hline Ganado & $0=$ no; $1=$ leve/poco frecuente; $2=$ sí & 1 & 2 & 1 & 1 & 0 & 2 & 2 \\
\hline Corte de césped & $0=$ no; $1=$ sí & 0 & 0 & 0 & 0 & 1 & 0 & 0 \\
\hline Total & & 1 & 4 & 3 & 4 & 5 & 2 & 2 \\
\hline
\end{tabular}

(BT: borde talar; BP: borde potrero; CI: cultivo con dosis reducidas de pesticidas; CII: cultivo con dosis convencionales de pesticidas; JA: jardín parquizado; PO: potrero; TA: talar).

(BT: tala forest edge; BP: pasture edge; CI: crop with low doses of pesticides; CII: crop with conventional doses of pesticides; JA: garden; PO: pasture; TA: tala forest).

doméstico disueltas en $10 \mathrm{~L}$ de agua. Las trampas fueron cubiertas parcialmente con elementos circundantes (i.e., trozos de ramas) propios del sistema para disminuir la desecación por insolación, la caída de agua de lluvia o de hojarasca en su interior. El recambio del contenido se realizó mensualmente desde el 10 de diciembre de 2010 al 19 de diciembre de 2011.

Además de los individuos adultos se registraron larvas pertenecientes a siete especies distribuidas en sendos géneros. Su identificación, excepto en los casos de S. anthracinus y Bradycellus viduus, que fueron criadas con anterioridad en nuestro laboratorio a partir de posturas de hembras grávidas mantenidas durante todo su ciclo vital, no estuvo exenta de dificultades. Con el propósito de lograr la mejor aproximación posible a su identidad específica, se procedió del siguiente modo. Primero, nos aseguramos su correcta asignación tribal preparando las larvas según el método aconsejado por Bousquet y Goulet (1984), y luego utilizamos las claves generales disponibles para larvas hasta nivel de tribu y género (Van Emden 1942; Thompson 1979; Makarow 1994; Costa et al. 1988). Una vez conseguido esto (tomando como aceptablemente bueno el consenso conseguido en dos de estos trabajos) se procedió a asociarlas con los representantes de esos géneros hallados conjuntamente con las larvas en la misma trampa. Este procedimiento se aplicó en tres casos particulares: las larvas de Selenophorus, Anisostichus y Pelmatellus, que a su vez presentan una relación de tamaño larva III-adulto diferente entre cada una de ellas (lo que permitió realizar la asociación con mayor seguridad). Consideramos que este procedimiento es razonable hasta tanto no se cuente con más estudios taxonómicos de las larvas de estas tribus.

\section{Análisis de los datos}

Las distintas especies tienen parámetros de captura propios, como el patrón de actividad, los hábitos de vida y la capacidad de ser capturadas ("catchability") por el método aquí empleado. Por eso, es recomendable reescalar las capturas por especie a través de todos los sitios de muestreo (Turin et al. 1991; Kotze et al. 2011). Para ello, primero se suma para cada especie la cantidad de especímenes capturados por trampa en un año. Por contingencias tales como la desecación, el pisoteo o la pérdida de trampas, no todas las trampas estuvieron funcionando todo el tiempo durante los 12 períodos de muestreo; entonces, el número total de especímenes por trampa fue dividido por el número de días de actividad efectivos y luego multiplicado por 365 para obtener una estimación del número de especímenes por especie capturado en un año (abundancia estandarizada). Posteriormente, esta estimación fue reescalada para cada una de las especies. Esto consistió en asignar el 100\% al valor más alto de abundancia de una especie para luego recalcular, en función de esto, el 
porcentaje de especímenes por año en los otros ambientes para esa misma especie. Estas estimaciones designadas como "presencias relativas" son los datos que se calcularon por trampa y se utilizaron para realizar el análisis de similitud más abajo mencionado, dado que permiten comparaciones entre especies con distintos hábitos a través de una serie de muestras (Kotze et al. 2011).

\section{Riqueza específica}

La riqueza específica se analizó mediante la construcción de curvas de rarefacción basadas en individuos, para comparar entre los valores de riqueza alfa acumulada (Colwell et al. 2004). La diversidad alfa acumulada es el número de especies que se recolecta en un punto o lugar (i.e., la extensión mínima en términos de espacio y tiempo que contiene una muestra del ensamble) en un determinado lapso (Halffter and Moreno 2005) (en este caso, en un año). La significancia estadística $(P<0.05)$ para la diferencia en la riqueza de especies entre los ambientes fue evaluada mediante la comparación de los límites de los intervalos de confianza del $95 \%$ en un punto con el mismo número de individuos en las curvas de rarefacción.

\section{Actividad}

Para analizar posibles diferencias entre el número total de individuos, tomado como medida de la actividad anual registrada durante todo el período de estudio en cada trampa, entre los distintos ambientes se realizaron pruebas de Kruskal-Wallis. Para saber entre qué pares de ambientes hubo diferencias se realizaron análisis de comparaciones múltiples. Para estos análisis se utilizó el programa R versión 2.13.2 (Bates et al. 2008).

\section{Composición y estructura del ensamble}

Para comparar la composición y estructura de los ensambles de los distintos ambientes se realizaron análisis de escalamiento multidimensional no métrico (MDS) y análisis de similitud (ANOSIM) en función de las matrices de similitud de Bray-Curtis, que trabajan con aspectos cuantitativos de los datos. El MDS reconstruye un mapa de las muestras en dos (o más) dimensiones, en el cual la distancia relativa entre muestras refleja la similitud relativa entre la composición de especies. El valor de "stress" indica cuánto se corresponde la representación espacial de las muestras en el gráfico con los rangos de similitud original. Por su parte, el ANOSIM es un procedimiento de permutación aplicado a matrices de rango de similitud (o disimilitud); utiliza el estadístico $R$, que es una medida comparativa del grado de separación entre ambientes. Si $R=1$, las repeticiones dentro de un ambiente son más similares entre sí que con cualquier repetición de ambientes distintos. Por su parte, $\mathrm{R}$ es igual a 0 si la hipótesis nula es cierta, es decir que las similitudes entre hábitats y dentro de hábitats son las mismas, en promedio (Clarke 1993).

\section{Potenciales especies o grupos de especies típicas de los ambientes}

Una especie es típica de un ambiente si el número de individuos recolectados es consistente en todas las muestras de ese ambiente (Clarke 1993). La contribución de cada especie a la similitud dentro de cada grupo (ambiente) y, en consecuencia, la evaluación de la potencialidad de estas como especies típicas, se analizó a través de porcentajes de similitud (SIMPER). Cuanto más abundante es una especie dentro de un ambiente, mayor es su contribución a las similitudes intra-ambiente.

Los análisis de ANOSIM, MDS y SIMPER se realizaron con el programa PRIMER 5 (Clarke and Gorley 2001). A su vez, se indicó el principal período de actividad y el pico de máxima actividad de las especies típicas identificadas por el análisis de SIMPER, según el método de los cuartiles propuesto por Fazekas et al. (1997). El método registra el pico de máxima actividad como la fecha en la que se registró el 50\% de la actividad. El comienzo y el final del principal período de actividad fueron definidos como las fechas en las que se registró el 25\% y el 75\% de la actividad.

\section{Asociación estacional de las especies más activas} de carábidos con la vegetación

Se utilizó el análisis de correspondencia canónico (CCA) para encontrar relaciones por estación entre la abundancia estandarizada de las especies y el porcentaje de cobertura vegetal de las familias de herbáceas. Las familias de herbáceas fueron las variables incluidas para cada estación del año. La cobertura vegetal se midió a partir de un aro metálico de $1 \mathrm{~m}$ de diámetro. Se tomaron tres medidas dentro de cada ambiente una vez por estación. Las significancias de cada ordenamiento y de las variables fueron puestas 
a prueba mediante análisis de permutaciones de Monte Carlo, usando el estadístico F (Legendre and Legendre 1998). Los análisis fueron realizados con el programa $R$ (Bates et al. 2008). La identificación de las plantas se realizó mediante bibliografía (Montes et al. 2007; Nisensohn et al. 2009; Faccini et al. 2008) y con la colaboración de la botánica María Celia Nuciari.

\section{Proporción de eurítopos, macrópteros y sinantrópicos}

Las especies fueron clasificadas de acuerdo con la bibliografía previa: Cicchino (2003; 2006a,b), Cicchino y Farina(2005), Caneppuccia et al. (2009), Marasas et al. (2010), Nanni (2010) en eurítopos y estenótopos y ubicuistas según el número y la tipología del/los hábitats que frecuentan. Estos términos fueron explicados por Cicchino (2003) y por Rainio y Niemelä (2003), y definen a las especies estenótopas como aquellas que frecuentan un único biotopo o un número reducido de ellos, que comparten condiciones microambientales de humedad, edáficas y / o florísticas similares. Por otra parte, las especies eurítopas son aquellas que viven en un amplio rango de biotopos, mientras que las especies ubicuistas son aquellas con amplia distribución geográfica, que a su vez pueden ser eurítopas o estenótopas. A su vez, las especies se clasificaron en sinantrópicas y no sinantrópicas (según su asociación directa o no con las viviendas humanas) y en macrópteras, pteridimórficas y ápteras o braquípteras según la morfología alar. Luego, a partir de las abundancias estandarizadas se comparó el porcentaje de individuos pertenecientes a cada categoría a través de los distintos ambientes mediante análisis de Kruskal-Wallis. Para identificar entre qué pares de ambientes hubo diferencias se realizaron análisis de comparaciones múltiples.

\section{Resultados}

Los ambientes fueron clasificados según el grado de modificación antrópica (Tabla 1). Con este criterio se identificaron cinco niveles de modificación en los cuales los ambientes se ordenaron de mayor a menor intensidad: 1) jardín parquizado, 2) cultivo CII y borde potrero, 3) cultivo $\mathrm{CI}, 4)$ potrero y talar, y 5) borde del talar.

\section{Riqueza específica}

En un año de muestreo se censaron 1751 individuos pertenecientes a 51 especies, distribuidas en 29 géneros. Al punto de rarefacción de $\mathrm{n}=101$ individuos, los valores de riqueza específica (S) más elevados los presentaron los ambientes de jardín, talar y borde del talar, seguidos por los valores decrecientes de los cultivos, el borde del potrero y el potrero (Tabla 2).

\section{Actividad anual}

Se detectaron diferencias en la abundanciaactividad de carábidos entre los ambientes $(\mathrm{H}=27.95, \mathrm{df}=6, P<0.001)$. El borde de potrero fue el ambiente cuya actividad fue más alta y variable. La prueba de comparaciones múltiples detectó que la actividad en el borde del potrero (mediana=128.8) fue significativamente más alta que en el jardín (mediana=20.6; $\mathrm{H}=31.08 ; P<0.05$ ) y que en los cultivos CI (mediana=27.8; $\mathrm{H}=22.58 ; P<0.05$ ) y CII (mediana=25.6; $\mathrm{H}=25.75 ; P<0.05$ ). A su vez, la actividad en el borde del talar (mediana=67.2) fue mayor que la actividad en el jardín $(\mathrm{H}=24.25 ; \mathrm{P}<0.05)$.

\section{Composición y estructura del ensamble.}

Los resultados muestran que los ensambles de carábidos se agruparon más en función de la estructura del hábitat que según su

Tabla 2. Riqueza específica (S) según el análisis de rarefacción a n=101.

Table 2. Species richness (S) according to an analysis of rarefaction with $\mathrm{n}=101$.

\begin{tabular}{lcccccc}
\hline \multicolumn{1}{c}{ Ambiente } & $\begin{array}{c}\text { Estructura } \\
\text { ambiente }\end{array}$ & $\begin{array}{c}\mathrm{N}^{\mathrm{o}} \text { total de } \\
\text { individuos }\end{array}$ & $\begin{array}{c}\mathrm{S} \text { (mediana) a } \\
\mathrm{n}=101\end{array}$ & $\mathrm{LS}$ & $\mathrm{LI}$ & $\begin{array}{c}\text { Diferencias } \\
\text { significativas }\end{array}$ \\
\hline Jardín & mixto & 109 & 25 & 26 & 24 & $\mathrm{a}$ \\
Talar & bosque & 213 & 22 & 26 & 18 & $\mathrm{a}, \mathrm{b}$ \\
Borde del talar & bosque & 350 & 22 & 25 & 19 & $\mathrm{a}, \mathrm{b}$, \\
Cultivo I & abierto & 135 & 18 & 19 & 16 & $\mathrm{~b}, \mathrm{c}$ \\
Cultivo II & abierto & 101 & 15 & 15 & 15 & $\mathrm{~d}$ \\
Borde del potrero & bosque & 704 & 12 & 16 & 9 & $\mathrm{c}, \mathrm{d}, \mathrm{e}$ \\
Potrero & abierto & 139 & 12 & 12 & 9 & $\mathrm{e}$ \\
\hline
\end{tabular}

Letras distintas indican diferencias significativas $(P<0.05)$. (LS: límite superior del intervalo de $95 \%$ de confianza; LI: límite inferior del intervalo de $95 \%$ de confianza).

Different letters indicate significant differences $(P<0.05)$. (LS: upper limit of the 95\% confidence; LI: lower limit of the $95 \%$ confidence). 
intensidad de modificación antrópica, aunque el stress resultó ligeramente elevado (0.17, en tres dimensiones). En la Figura 2 puede interpretarse que el eje $x$ representa un gradiente desde los ambientes abiertos hacia los ambientes de bosque. El grupo de ambientes abiertos incluye al potrero y los cultivos, mientras que el grupo de ambientes

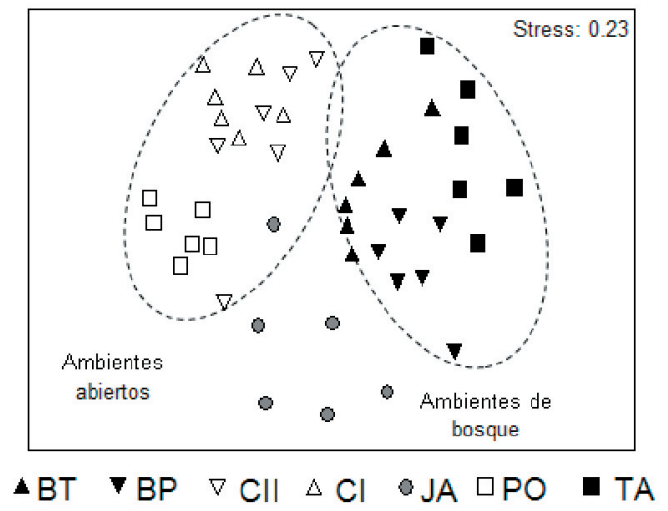

Figura 2. Análisis de MDS de los ensambles de carábidos en los distintos ambientes. En color negro se representaron los ambientes de bosque; en color blanco, los ambientes abiertos y en color gris el ambiente del jardín, parcialmente forestado. Para mayor claridad se muestra el gráfico en dos dimensiones. BP: borde potrero; BT: borde talar; $\mathrm{CI}$ : cultivo I; CII: cultivo II; JA: jardín parquizado; TA: talar centenario; PO: potrero.

Figure 2. MDS analysis of carabid assemblages in different environments. The graph is shown in two dimensions for greater clarity. Black symbols: forest environments; white symbols: open environments; grey symbols: garden, partly forested. BT: tala forest edge; BP: pasture edge; CI: crop with low doses of pesticides; CII: crop with conventional doses of pesticides; JA: garden; PO: grazing pasture area; TA: tala forest.

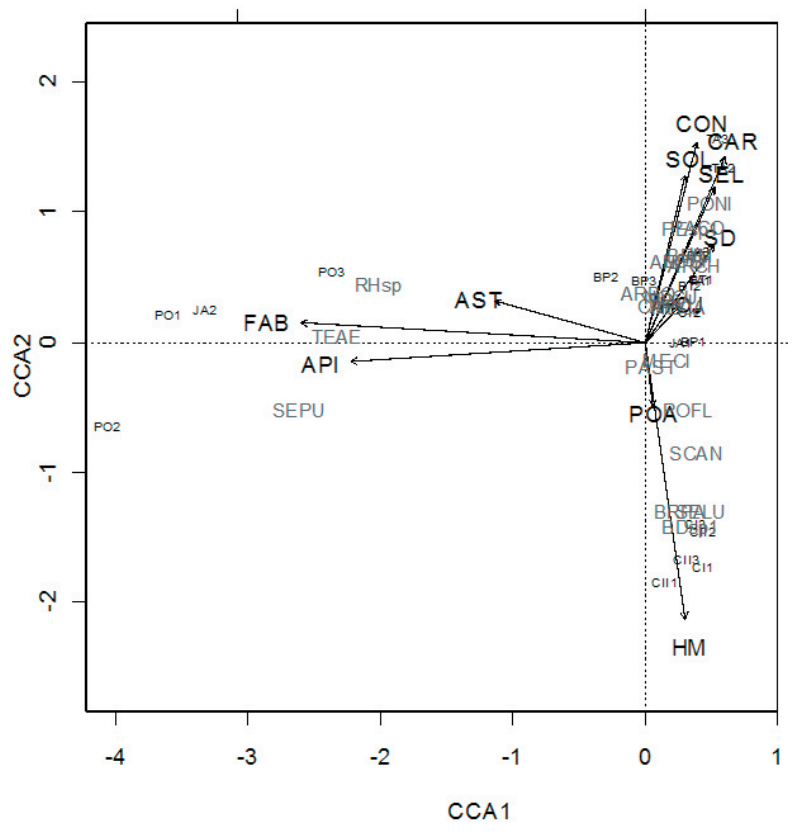

de bosque incluye al talar y a los bordes. El jardín se ubica entre medio de ambos grupos. El análisis de ANOSIM mostró que hay diferencias entre los ensambles de todos los ambientes $(\mathrm{R}=0.693, P=0.001)$.

\section{Asociación estacional de las especies más activas de carábidos con la vegetación}

Los análisis de CCA revelaron que las especies de carábidos consideradas no están relacionadas con el porcentaje de cobertura de las familias de herbáceas en invierno (prueba de permutación de Monte-Carlo: $\mathrm{F}=1.63$, $P=0.25$ ), otoño (prueba de permutación de Monte-Carlo: $\mathrm{F}=0.99, P=0.79)$ y verano (prueba de permutación de Monte-Carlo: $\mathrm{F}=0.97, P=0.63)$. La prueba indicó que sólo en primavera la cobertura de herbáceas estuvo asociada al ensamble de carábidos (prueba de permutación de Monte-Carlo: $\mathrm{F}=1.31, P=0.02)$. La inercia total del modelo fue 3.777 y los autovalores restringidos para los ejes CCA1 y CCA2 fueron 0.617 y 0.487 , respectivamente. Sólo los primeros dos ejes canónicos fueron significativos (eje 1: $\mathrm{F}=3.84 ; P<0.05$; eje 2 : $\mathrm{F}=3.02 ; P<0.05)$. Las variables que resultaron significativas fueron: Apiaceae (prueba de permutación de MonteCarlo: $F=2.23, P=0.02$ ), Fabaceae (prueba de permutación de Monte-Carlo: $\mathrm{F}=1.91, P=0.02)$ y Convolvulaceae (prueba de permutación de Monte-Carlo: $\mathrm{F}=1.91, P=0.007$ ) (Figura 3). Las especies de carábidos asociadas a Fabaceae y Apiaceae fueron Selenophorus punctulatus, Rhytidognathus sp. y Tetragonoderus aeneus. Las especies de carábidos con una asociación más

Figura 3. Biplot de la presencia relativa de carábidos y las familias de herbáceas (flechas) a partir del CCA. Los nombres abreviados de las especies están representados por cuatro letras (se aclaran sólo abreviaturas de especies con asociación significativa. SEPU: Selenophorus punctulatus, TEAE: Tetragonoderus aeneus, RHsp: Rhytidognathus sp., PONI: Polpochila nigra). Las abreviaturas de las familias de herbáceas están representados por tres letras al extremo de cada flecha (POA: Poaceae; API: Apiceae; FAB: Fabaceae; AST: Asteraceae; SOL: Solaneceae; SEL: Selaginellaceae; CAR: Caryophyllaceae; CON: Convolvulaceae; HOJ: hojarasca; SD: suelo desnudo; HM: hoja de maíz). Los ambientes muestreados están abreviados con dos letras (ver referencias en la Figura 1) y un número.

Figure 3. Biplot for the relative presence of carabids and plants families (arrows) obtained by CCA. The abbreviated names of species are represented by four letters (only abbreviations of some species with significant association are clarified: SEPU: Selenophorus punctulatus, TEAE: Tetragonoderus aeneus, RHsp: Rhytidognathus sp.; PONI: Polpochila nigra). The abbreviated names of the herbaceous plants families are represented by three letters at the end of each arrow (POA: Poaceae; API: Apiceae; FAB: Fabaceae; AST: Asteraceae; SOL: Solaneceae; SEL: Selaginellaceae; CAR: Caryophyllaceae; CON: Convolvulaceae; HOJ: litter; $\mathrm{SD}=$ bare soil; $\mathrm{HM}=$ maize leaf). Sampled environments are abbreviated to two letters (see references in Figure 1) and a number. 
fuerte con las Convolvulaceae fueron Polpochila nigra, Paranortes cordicollis, Pelmatellus sp. №1, Brachinus immarginatus, Loxandrus simplex, Anisostichus posticus y Argutoridius chilensis.

\section{Proporción de eurítopos, macrópteros y sinantrópicos}

Eurítopos. La prueba de Kruskal-Wallis no arrojó diferencias estadísticamente significativas en el porcentaje de individuos pertenecientes a especies eurítopas entre los distintos ambientes $(\mathrm{H}=4.99, \mathrm{df}=6, \mathrm{P}=0.54)$.

Macrópteros. Hubo diferencias en el porcentaje de individuos pertenecientes a especies macrópteras entre los distintos ambientes $(\mathrm{H}=26.82, \mathrm{df}=6, P=0.0001)$. Los ambientes con porcentajes más altos de macrópteros fueron los cultivos (CI y CII) y el potrero (PO), con medianas: $96.5 \%$ en $\mathrm{CI}$, $94.1 \%$ en CII y $94.6 \%$ en PO. De acuerdo con el análisis de comparaciones múltiples CI se diferenció significativamente del borde del talar (BT) y del talar (TA), con medianas: $71.8 \%$ en BT y $65.0 \%$ en TA $(\mathrm{H}=22.00, P<0.05$ y $\mathrm{H}=26.58, P<0.05$, respectivamente). El cultivo CII se diferenció del talar (TA) $(\mathrm{H}=23.25$, $P<0.05)$, y el potrero (PO), del talar (TA) $(\mathrm{H}=23.33, \mathrm{P}<0.05)$.

Sinantrópicos. Se detectaron diferencias en el porcentaje de individuos pertenecientes a especies sinantrópicas $(\mathrm{H}=13.53, \mathrm{df}=6, \mathrm{P}<0.05)$.
El análisis de comparaciones múltiples detectó diferencias significativas únicamente entre el cultivo CII (mediana $=86.4 \%$ ), con mayor porcentaje de sinantrópicas que el potrero (mediana $=52.5 \% ; \mathrm{H}=22.00 ; P<0.05$ ).

\section{Potenciales especies o grupos de especies típicas de los ambientes}

A partir de los resultados del SIMPER se distinguieron especies típicas de los ambientes abiertos. Estas especies fueron: Bradycellus sp. $\mathrm{N}^{\mathrm{o}} 1$, Polpochila flavipes, Rhytidognathus sp., Selenophorus lugubris, Selenophorus punctulatus, Scarites anthracinus y Cynthidia planodisca, con limitada presencia en los ambientes forestados. Pachymorphus striatulus apareció como especie típica de ambientes abiertos, pero también con importante actividad en los bordes, sobre todo en el borde del talar.

Por otro lado, las especies típicas de los ambientes de bosque incluyeron a: Metius circumfusus, Notiobia cupripennis, Argutoridius bonariensis, Carbonellia platensis, Anisostichus posticus, Plagioplatys vagans y Pelmatellus sp. №1. Sólo dos especies no fueron registradas fuera de estos tres ambientes de bosque: Pelmatellus sp. №1, que fue la única que se reveló como típica del ambiente del talar, y Anisostichus posticus. Las especies restantes mostraron alta actividad en las zonas de

Tabla 3. Resultado del análisis SIMPER de las especies que contribuyen a más del 10\% de la similitud observada dentro de cada ambiente. Se indican los meses de registro del pico de máxima actividad y del principal período de actividad.

Table 3. Results of SIMPER analysis. Species that contribute to over $10 \%$ of the observed similarity within each environment. Months with activity peak and the main peak activity period are indicated.

\begin{tabular}{|c|c|c|c|c|c|c|c|c|c|}
\hline Especie & BT & $\mathrm{TA}$ & $\mathrm{BP}$ & $\mathrm{CI}$ & CII & $\mathrm{PO}$ & JA & $\begin{array}{c}\text { Pico de } \\
\text { actividad }\end{array}$ & $\begin{array}{l}\text { Principal } \\
\text { período } \\
\text { actividad }\end{array}$ \\
\hline Metius circumfusus & 13.24 & & & & & & & nov & nov \\
\hline Notiobia cupripennis & 12.78 & & & & & & 29.84 & ene & nov-mar \\
\hline Pachymorphus striatulus & 12.13 & & & & 20.11 & 37.40 & & abr & mar-jul \\
\hline Argutoridius bonariensis & & & 26.60 & & & & & abr & dic-jun \\
\hline Carbonellia platensis & & & 17.56 & & & & & dic & nov-dic \\
\hline Anisostichus posticus & & & 12.34 & & & & & dic & nov-ene \\
\hline Plagioplatys vagans & & & 11.54 & & & & & mar & feb-abr \\
\hline Bradycellus sp. №1 & & & & & 24.81 & & & nov & nov-dic \\
\hline Polpochila flavipes & & & & 12.08 & 19.92 & & & nov & nov \\
\hline Rhytidognathus sp. & & & & & 11.80 & 18.59 & & may & abr-jun \\
\hline Selenophorus lugubris & & & & 26.00 & & & & dic & nov-ene \\
\hline Scarites anthracinus & & & & 25.93 & & & & nov & nov-dic \\
\hline Cynthidia planodisca & & & & 15.66 & & 18.62 & & feb & feb \\
\hline Pelmatellus egenus & & & & & & & 19.97 & dic & dic \\
\hline Selenophorus punctulatus & & & & & & 19.55 & & nov & oct-dic \\
\hline Pelmatellus sp. № 1 & & 31.08 & & & & & & nov & oct-dic \\
\hline
\end{tabular}

(BT: borde talar; BP: borde potrero; CI: cultivo con dosis reducidas de pesticidas; CII: cultivo con dosis convencionales de pesticidas; JA: jardín parquizado; PO: potrero; TA: talar).

(BT: tala forest edge; BP: pasture edge; CI: crop with low doses of pesticides; CII: crop with conventional doses of pesticides; JA: garden; PO: grazing pasture area; TA: tala forest). 
bordes, con presencia en mayor o menor grado hacia los ambientes abiertos.

Por último, dos especies típicas fueron identificadas para el ambiente del jardín parquizado: N. cupripennis, también presente en alto número en el borde del talar, y Pelmatellus egenus, ambas especies eurítopas.

En cuanto a la actividad la gran mayoría de estas especies manifestaron su pico de actividad en primavera (noviembre) y verano (diciembre-febrero), mientras que sólo tres lo hicieron en verano tardío (marzo) o en otoño (abril-mayo) (Tabla 3).

\section{Presencia de larvas}

Todas las larvas registradas fueron recolectadas en verano. No se registraron larvas en el potrero ni en el cultivo CII. A continuación se enumeran las especies o géneros de larvas registradas en distintos ambientes, con el número de individuos escrito entre paréntesis.

Borde del talar. Anisostichus posticus (1), Bradycellus viduus (1), Galerita collaris (16) y Polpochila sp. (4).

Borde del potrero. A. posticus (1), B. viduus (3), G. collaris (3)

Cultivo CI. Polpochila sp. (1) y Selenophorus sp. (1)

Jardín. G. collaris (2) y S. anthracinus (1)

Talar. G. collaris (2) y Pelmatellus sp (1)

\section{Discusión}

A partir de nuestros resultados podemos inferir que el uso del suelo y la estructura del ambiente influyen sobre la diversidad de carábidos a escala local. A pesar de que algunas especies de carábidos tengan la potencialidad de desplazarse grandes distancias en un día (Thiele 1977), el tipo de ambiente y su uso son determinantes para las especies que lo frecuentan. El diseño permitió detectar diferencias en los ensambles entre los distintos ambientes estudiados, aunque debido a las características del paisaje no se pudo contar con repeticiones de los ambientes muestreados. De todos modos, la información obtenida es muy valiosa y constituye un muy buen contexto para explicar la actividad del ensamble de este talar, sobre todo porque hay patrones en la afinidad de hábitat de las especies que concuerdan con estudios previos realizados en la Provincia de Buenos Aires.

En el agroecosistema estudiado se observa que hay una tendencia a que la estructura de los ambientes, más que el grado de modificación antrópica, afecte la riqueza específica y la composición y estructura de los ensambles. Hay una tendencia marcada a encontrar valores de riqueza más altos en los ambientes de bosque en comparación con los ambientes abiertos, independientemente de su grado de modificación. La excepción fue el borde del potrero, compuesto por talas y cipreses, que presentó valores muy bajos de riqueza, junto con los ambientes abiertos como el área cultivada con manejo convencional (cultivo CII) y el potrero de pastoreo. El jardín parquizado alberga una riqueza elevada de carábidos, a pesar de atravesar modificaciones cíclicas como el corte de césped y el riego. Es seguro que la riqueza registrada responda a que el jardín se encuentra rodeado por el talar, a la vez que cuenta con una mayor oferta de microambientes que emergen de la acumulación de escombros, grietas o refugios provistos por las edificaciones (Cicchino 2003). A su vez, cuenta con parches amplios de Dichondra sp. (Convolvulaceae) que retienen un importante gradiente de humedad edáfica, en adición a la heterogeneidad en la composición y estructura de la vegetación arbustiva y arbórea que favorecen la permanencia de mayor diversidad de especies de carábidos. Las actividades cíclicas que se operan de manera periódica en este ambiente le confieren características de un ambiente estructuralmente inestable, y se comporta como una comunidad seral en desarrollo que nunca llega a un punto de estabilidad, por lo que su carga especifica puede ser mayor que la esperada (Connel 1978). A diferencia del jardín, el talar es utilizado como zona de descanso y pastoreo para el ganado con la misma frecuencia e intensidad que el potrero. El borde del talar se encuentra sometido al mismo tipo de uso de suelo, aunque la presencia de un borde de alambrada restringe el tránsito del ganado y permite un mayor desarrollo de la vegetación herbácea. El denso dosel de la vegetación arbórea permanente en estos ambientes es la principal causa de que las fluctuaciones diurnas de temperatura sean reducidas en comparación con los terrenos abiertos y, de esa manera, las tasas de mortalidad de los artrópodos en la superficie del suelo son menores (Pfiffner and Luka 2000), lo que facilita la actividad y permanencia de especies que requieren condiciones de hábitats más estables y/o hidrófilas. 
También cabe la posibilidad de que el criterio de clasificación de los ambientes aquí propuesto para el grado de modificación antrópica no se corresponda en su totalidad con la percepción de este desde la perspectiva de la comunidad de carábidos. Conforme con esto, podrían existir otras variables que modifiquen con distinta intensidad los ambientes abiertos y que no fueron consideradas, o que alguna de las variables consideradas tuviese un mayor impacto que el que arbitrariamente le fuera asignado por nosotros. No obstante, las diferencias entre los ensambles de los ambientes de bosque y de los ambientes abiertos pueden ser explicadas por las preferencias de hábitat de las especies particulares de los carábidos. Los porcentajes más altos de especies macrópteras los presentaron los ambientes abiertos: los cultivos y el potrero. En los bosques boreales se conoce que las especies especialistas de bosques generalmente son no voladoras y presentan alas reducidas, por lo que es probable que su movilidad sea dificultosa a través de los pastizales o praderas para trasladarse de un parche a otro de bosque (Niemelä 2001). A su vez, las alas reducidas han sido propuestas como una adaptación de las poblaciones estables y duraderas a sus ambientes (Venn 2007). En los ambientes de Laguna Nahuel Rucá, las especies pteridimórficas fueron más frecuentes en los ambientes de bosque: Bradycellus viduus, Pelmatellus sp. $\mathrm{N}^{\mathrm{o}} 1$, Paranortes cordicollis y Carbonellia platensis (en cuyas poblaciones del sudeste bonaerense sólo se han registrado individuos micrópteros de esta última especie). Incluso, la proporción de individuos macrópteros registrada dentro del talar para las poblaciones de Bradycellus viduus (Cicchino et al. 2011) y Paranortes cordicollis (Porrini et al. 2010) no supera el 10\%. Las especies macrópteras y voladoras son capaces de utilizar los ambientes abiertos (Venn et al. 2003) además de ser buenas colonizadoras en ambientes disturbados o inestables (Venn 2007). Sin embargo, la única especie áptera (Rhytidognathus sp.) fue muy frecuente en los cultivos y el potrero, como discutiremos más abajo.

Los bordes de bosques tienen una gran importancia en el manejo ambiental, ya que permiten que funcionen como fuente de carábidos con el potencial de dispersarse y recolonizar los ambientes modificados por las actividades humanas (Magura 2002). A su vez, funcionan como refugios durante la hibernación e, incluso, como sitios donde es posible la reproducción (Desender and Alderweireldt 1988), como lo ha demostrado la presencia de larvas que se han registrado en estos ambientes. Cabe destacar que de las 25 especies censadas en los ambientes abiertos, 22 también estuvieron presentes en los bordes o en el talar. Por ejemplo, Argutoridius bonariensis, una especie eurítopa y ubicuista, está presente en ambientes tales como bosques de tala (Cicchino 2006a,b), parches de Myoporum laetum (siempreverde) y de Arundo donax (caña de castilla) (Marasas et al. 2010), en viñedos (Paleologos 2011), en huertas y quintas de producción hortícola (Porrini et al. 2015), en ambientes citadinos (Cicchino 2003, 2010; Farina and Cicchino 2011), entre otros y, en nuestro estudio, en los bordes arbolados. Pero tiene la capacidad de colonizar sitios altamente disturbados como terrenos de pastoreo (Canepuccia et al. 2009), áreas cultivadas (Sorensen 2006; Turienzo 2006; Marasas et al. 2010) o sitios afectados por incendios (Cicchino 2006a). Estos resultados coinciden con trabajos previos, en los que se demostró que la riqueza de especies de carábidos (Cividanes and dos SantosCividantes 2008) y su actividad (Pfiffner and Luka 2000; Marasas et al. 2010) son elevadas en los márgenes de los lotes cultivados. Los bordes, que no están arados o disturbados, funcionan como sitios de hibernación y/o refugio para muchos artrópodos, carábidos incluidos (Marasas 2012).

Como se mencionó antes, las larvas fueron registradas en los ambientes asociados al tala, el único ambiente abierto en el que se registraron dos larvas fue en el cultivo en el que el uso de agroquímicos fue reducido. La distribución de las larvas a su vez no sólo depende de la disponibilidad de alimento, sino de las condiciones del suelo y el mantillo que favorezcan la humedad (Kagawa and Maeto 2009). Las larvas de Anisosticus, Bradycellus, Selenophorus y Polpochila comparten los hábitos básicamente seminívoros con los adultos, de la misma manera que las larvas y los adultos de Scarites y Galerita son ambos marcadamente depredadores. Este registro de larvas de especies de variados tamaños y hábitos nos indica que en estos sitios existe una oferta de alimento, tanto de presas animales como de calidad y tamaño de semillas, que permiten su supervivencia y crecimiento. Las larvas de Galerita se caracterizan por sus hábitos gregarios, por ser ágiles patrulladoras crepusculares o nocturnas, capaces de recorrer grandes distancias y atravesar obstáculos. 
Las larvas seminívoras también muestran actividad crepuscular o nocturna, aunque su desplazamiento es notablemente más lento debido a su conformación corporal. Estos resultados muestran que la conservación de parches de ambientes seminaturales en los agroecosistemas es primordial para la reproducción de muchas especies de carábidos y que sus propiedades amortiguadoras pueden constituir un factor clave en la selección de sitios de hibernación, que a su vez incrementaría el número y las especies de artrópodos benéficos que visitan los campos arables (Pfiffner and Luka 2000; Marasas et al. 2010).

La actividad total de los carábidos mostró una tendencia marcada a ser mayor en los ambientes de borde, sobre todo en el borde del potrero. Esto, junto con la riqueza específica, revela una baja diversidad en este borde, indicando que unas pocas especies se trasladan con frecuencia por ese corredor, como por ejemplo A. bonariensis y C. platensis. Es probable que la hojarasca generada por el talar y por los cipreses, creen condiciones de temperatura y humedad (Koivula et al. 1999) y una estructura que favorezca el desplazamiento de unas pocas especies de pequeña talla como las anteriormente mencionadas, y dificulte el tránsito de otras especies de tallas mayores, en particular durante el período otoñal e invernal.

El estudio de las preferencias de hábitat de las especies individuales es una herramienta aún más completa para el análisis de la biodiversidad (Niemelä 2000). Una sola especie, Pelmatellus sp. $\mathrm{N}^{\mathrm{o}} 1$, se reveló como típica del talar, mientras que otras, como $M$. circumfusus, $A$. bonariensis, C. platensis, A. posticus, y $P$. vagans predominaron en los bordes arbolados y mostraron un grado de asociación variable con el tapiz vegetal de Dichondra sp. (Convolvulaceae), predominante en dichos ambientes. En los ambientes abiertos, como los cultivos y el potrero, las especies predominantes fueron $P$. striatulus, S. lugubris, S. anthracinus, Bradycellus sp. $\mathrm{N}^{\circ} 1$, P. flavipes, C. planodisca, S. punctulatus y Rhytidognathus sp. Estas dos últimas también mostraron una asociación fuerte con plantas herbáceas de las familias Apiaceae y Fabaceae, muy abundantes sobre todo en el potrero de pastoreo y en las áreas abiertas del jardín parquizado, sin que hasta el momento puedan explicarse con seguridad las razones de esta afinidad florística.
En las áreas cultivadas, las especies muy susceptibles tienden a desaparecer y aquellas que frecuentan de forma permanente estos ambientes están adaptadas a los disturbios repetidos productodelas actividades decultivo (Huusela-Veistola 1996). Es esperable que las especies eurítopas sean dominantes en los ambientes más disturbados y/o simplificados y que la diversidad en ellos sea más baja (Jung et al. 2012). No obstante, no se detectaron diferencias en el porcentaje de eurítopos entre los distintos ambientes estudiados, aunque el porcentaje de sinantrópicos fue alto en el cultivo con manejo convencional. Entre las especies que se consideran eurítopas, están aquellas presentes en una muy amplia variedad de hábitats, como, por ejemplo, P. striatulus, S. anthracinus, Bradycellus sp. $\mathrm{N}^{\mathrm{o}} 1$, que son componentes frecuentes de la fauna de carábidos en los agroecosistemas del centro este de la Argentina (Marasas et al. 2001, 2010; Sorensen 2006; Turienzo 2006; Lietti et al. 2008; Paleologos 2011). Otras especies son más especializadas y requieren ciertas características del hábitat. Por ejemplo, C. planodisca, S. punctulatus, S. lugubris, P. flavipes son consideradas estenótopas. Las dos primeras son frecuentes en ambientes xéricos (Sorensen 2006; Marasas et al. 2010; Paleologos 2011) y la última, más común en pastizales lindantes a cuerpos de agua (Paleologos 2011). No obstante, estas especies también están habitualmente presentes en agroecosistemas.

El caso de Rhytidognathus sp. es muy particular, ya que el género tiene una distribución geográfica muy restringida que se limita al Uruguay y, en la Argentina, a la localidad de La Plata (Roig-Juñent and Rouaux 2012) y al talar de Laguna Nahuel Rucá (Cicchino 2006b), en la Provincia de Buenos Aires. Rhytidognathus platensis fue registrada en los cultivos hortícolas con manejo orgánico, pero su hábitat original se asocia a ambientes seminaturales como los talares y a las áreas ribereñas (Roig-Juñent and Rouaux 2012), mientras que la especie registrada en el talar de Nahuel Rucá probablemente sea una especie diferente, aunque parece comportarse de manera similar, ya que según nuestros resultados fue muy activa en las áreas cultivadas, pero también es frecuente dentro del bosque (Cicchino 2006a).

Por otra parte, el caso de Pelmatellus sp. №1, C. platensis, $P$. vagans, $M$. circumfusus y A. posticus, podría ser ejemplo de especies más sensibles a los disturbios ambientales. Cicchino y Farina (2005) mencionan que 
las primeras tres especies nunca fueron registradas en el entorno urbano marplatense por fuera de la Reserva Natural del Puerto y que sólo mantienen poblaciones estables en sitios menos modificados, como en las reservas naturales y otros sitios protegidos (Cicchino and Farina 2005). A su vez, Marasas et al. (2010) registraron la presencia de $M$. circumfusus en los bordes de laguna y cortaderales y no en las áreas cultivadas, fundamentalmente por tratarse de una especie francamente hidrófila.

En términos de conservación, las especies eurítopas y ubicuistas probablemente sobrevivan sin protección si la variedad de hábitats que usan se mantiene (Rainio and Niemelä 2003). Las especies más estenótopas o sensibles, con requerimientos de hábitat más específicos, son un foco más apropiado de conservación y es necesario profundizar los estudios de especificidad de hábitat de estas especies a escala regional para poder predecir cómo se verán afectadas por el avance de las actividades humanas, considerando que esta especificidad puede variar geográficamente, según el nivel de escala que se tome en consideración (Cicchino 2003).

Finalmente, la distribución temporal de la actividad de las especies también es un factor clave a tomar en cuenta en el manejo de un agroecosistema, dado que implica la época de reproducción de las poblaciones y, en consecuencia, su supervivencia. Las especies que se reproducen en el período de prácticas intensivas son las más afectadas (Rainio and Niemelä 2003). Debe considerarse el momento del año en que se realice cualquier tipo de monitoreo de la biodiversidad, dado que hay grupos de especies que muestran su principal período de actividad en distintas estaciones.
A su vez, nuestros resultados indicaron que, luego de un estudio anual, de las 16 especies que se revelaron como típicas de distintos ambientes sólo cuatro manifestaron su pico de actividad en marzo-mayo (otoño), mientras que las 12 especies restantes mostraron el pico en primavera tardía o verano. Esto indica que en las estaciones cálidas podemos contar con una mayor cantidad de especies activas con la potencialidad de aportar más información acerca de las características del ambiente que en otoño o invierno.

A modo de conclusión, cabe destacar que, según nuestros resultados, los ensambles de carábidos difirieron en todos los ambientes estudiados, llegando a identificarse especies potencialmente bioindicadoras. En la escala local, el uso del suelo y la estructura del ambiente son factores que influyen sobre la composición y estructura de los ensambles de carábidos, aun cuando los ambientes están cercanos y conectados entre sí. A su vez, un agroecosistema con una estructura en mosaico compuesta de arboledas, bordes de vegetación espontánea, cercos, humedales, etc. puede crear hábitats múltiples que proveen sitio de reproducción, alimentación y refugio para muchos carábidos y otros artrópodos benéficos.

Agradecimientos. Agradecemos a la agencia Neotropical Grassland Conservancy por la donación de equipamiento, al CONICET por la beca doctoral que motivó esta investigación. También a la familia Urrutia, propietarios del predio, por su buena predisposición, a D. Grandinetti, L. Peralta, J.Arcusa, P. Revainera, C. Luna, S. Altamirano y A. G. Martins por su colaboración en las tareas de campo, y a nuestras familias y E. Delfino por su constante apoyo y guía.

\section{REFERENCIAS}

Adis, J. 2002. Recommended sampling techniques. Pp. 555-576 en J. Adis (ed.). Amazonian Arachnida and Myriapoda. PENSOFT Publishers, Moscú, Rusia.

Altieri, M. A. 1999. The ecological role of biodiversity in agroecosystems. Agriculture, Ecosystems and Environment 74:19-31

Bates, R., J. Chambers, P. Dalgaard, S. Falcon, R. Gentleman, K. Hornik, S. Iacus, R. Ihaka, F. Leisch, U. Ligges, T. Lumley, M. Maechler, D. Murdoch, P. Murrel, M. Plummer, B. Ripley, D. Sarkar, D. Temple Lang, L. Tierney, and S. Urbanek. 2008. R: A language and environment for statistical computing. R Foundation for Statistical Computing, Vienna, Austria. URL: www.R-project.org.

Bilenca, D., M. Codesido, C. González Fischer, L. Pérez Carusi, E. Zufiaurre, and A. Abba. 2012. Impactos de la transformación agropecuaria sobre la biodiversidad en la provincia de Buenos Aires. Revista del Museo Argentino de Ciencias Naturales 14:189-198.

Bousquet, Y. M. and H. Goulet. 1984. Notation of primary setae and pores on larvae of Carabidae (Coleoptera: Adephaga). Canadian Journal of Zoology 62:573-588.

Canepuccia, A. D., A. C. Cicchino, A. Escalante, A. Novaro, and J. P. Isaach. 2009. Differential responses of marsh arthropods to rainfall-induced habitat loss. Zoological Studies 48:174-183.

Castro, A. V., D. P. Porrini, and A. C. Cicchino. 2014. Annual activity density of groundbeetles (Coleoptera: Carabidae) 
of a Celtis ehrenbergiana (Rosales: Celtidaceae) forest of Buenos Aires province, Argentina. Journal of Insect Science 14:1-14.

Cicchino, A. C., A. V. Castro, and D. P. Porrini. 2011. Rasgos biológicos y fenología estacional de Bradycellus viduus (Dejean, 1829) (Insecta, Coleoptera, Harpalini) en el sudeste de la provincia de Buenos Aires, Argentina. II Congreso Nacional de Ecología y Biología de Suelos, Mar del Plata, Buenos Aires.

Cicchino, A. C. 2003. La carabidofauna edáfica de los espacios verdes del ejido urbano y suburbano marplatense. Su importancia como herramienta de manejo de estos espacios. Revista de Ciencia y Tecnología 8:145-164.

Cicchino, A. C. 2006a. Diversidad de carábidos (Insecta, Coleoptera, Carabidae) de dos asociaciones de tala en la Laguna de Los Padres, partido de General Pueyrredón, provincia de Buenos Aires. Pp. 128-136 en E. Mérida and J. Athor (eds.). Talares bonaerenses y su conservación. Fundación de Historia Natural "Félix de Azara", Buenos Aires, Argentina.

Cicchino, A. C. 2006b. Diversidad de carábidos (Insecta, Coleoptera, Carabidae) de un talar joven de la laguna Nahuel Rucá, partido de Mar Chiquita, provincia de Buenos Aires. Pp. 137-145 en E. Mérida and J. Athor (eds.). Talares bonaerenses y su conservación. Fundación de Historia Natural "Félix de Azara", Buenos Aires, Argentina.

Cicchino, A. C. 2010. Los carábidos edáficos (Insecta, Coleoptera, Carabidae) de una vivienda urbana típica del gran La Plata, provincia de Buenos Aires, Argentina. Pp. 233-250 en Trabajos Completos. VII REBIOS, San Miguel de Tucumán, Tucumán, Argentina.

Cicchino, A. C., and J. L. Farina. 2005. Carabidofauna de los suelos lindantes con la laguna litoral de la Reserva Natural del Puerto de Mar del Plata, provincia de Buenos Aires, Argentina. Pp. 1-15 en Trabajos completos. V REBIOS, Área temática I, Comunidades Terrestres III, San Salvador de Jujuy, Argentina.

Cividanes, F. J., and T. M. dos Santos-Cividanes. 2008. Distribuição de Carabidae e Staphylinidae em agroecossistemas. Pesquisa Agropecuária Brasileira 43:157-162.

Clarke, K. R. 1993. Non-parametric multivariate analyses of changes in community structure. Australian Journal of Ecology 18:117-143.

Clarke, K. R., and R. N. Gorley. 2001. PRIMER v5: User Manual/Tutorial. Plymouth Marine Laboratory, Plymouth.

Colwell, R. K., C. X. Maon, and J. Chang. 2004. Interpolating, extrapolating, and comparing incidence based species accumulation curves. Ecology 85:2717-2727.

Connell, J. H. 1978. Diversity in tropical rain forests and coral reefs. Science 199:1302-1303.

Costa, C., S. A. Vanin, and S. A. Casari-Chen. 1988. Larvas de coleoptera do Brasil. Museu de Zoologia. Universidade de São Paulo, São Paulo, Brasil.

Desender, K., and M. Alderweireldt. 1988. Population dynamics of adult and larval Carabid beetles in a maize field and its boundary. Lournal of Applied Entomology 106:13-19.

Erwin, L., G. E. Ball, D. R. Whitehead and A. Halpern (eds.). Carabid Beetles: Their Evolution, Natural History, and Classification, Springer Netherlands.

Faccini, D., L. Nisensohn, E. Puricelli, D. Tuesca, and L. Allieri. 2008. Malezas frecuentes en los agroecosistemas de la región sojera núcleo. Parte I. Facultad de Ciencias agrarias, Universidad Nacional de Rosario. Dow AgroSciences. Segunda edición, Buenos Aires, Argentina.

Farina, J. L., and A. C. Cicchino. 2011. La RNPMdP: una visión entomológica. Pp. 189-242 en Reserva Natural del Puerto de Mar del Plata, un oasis urbano de vida silvestre. De Marco, S., L. Vega and P. Bellagamba (eds.). Universidad FASTA, Mar del Plata, Buenos Aires, Argentina.

Fazekas, J., F. Kádár, M. Sárospataki, and G. Lövei. 1997. Seasonal activity, age structure and egg production of the ground beetle Anisodactylus signatus (Coleoptera: Carabidae) in Hungary. European Journal of Entomology 94:473484.

Huusela-Veistola, E. 1996. Effects of pesticide use and cultivation techniques on ground beetles (Col., Carabidae) in cereal fields. Annales Zoologici Fennici 33:197-205.

Jung, J., S. Kim, S. Lee, C. Park, E. Lee, and J. Lee. 2012. Ground beetle (Coleoptera: Carabidae) assemblage in the urban landscape. Korea Lournal of Ecology and Field Biology 35(2):79-89.

Kagawa, Y., and K. Maeto. 2009. Spatial population structure of the predatory ground beetle Carabus yaconinus (Coleoptera: Carabidae) in the mixed farmland-woodland satoyama landscape of Japan. European Journal of Entomology 106:385-391.

Koivula, M., P. Punttila, Y. Haila, and J. Niemelä. 1999. Leaf litter and the small-scaled distribution of carabid beetles (Coleoptera, Carabidae) in the boreal forest. Ecography 22:424-435.

Kotze D. J., P. Brandmayr, A. Casale, E. Dauffy-Richard, W. Dekoninck, M. J. Koivula, G. L. Lövei, D. Mossakowski, J. Noordijk, W. Paarmann, R. Pizzolotto, P. Saska, A. Schwerk, J. Serrano, J. Szyszko, A. Taboada, H. Turin, S. Venn, R. Vermeulen, and T. Zetto. 2011. Forty years of carabid beetle research in Europe: from taxonomy, biology, ecology and population studies to bioindication, habitat assessment and conservation. ZooKeys 100:55-148.

Legendre, P., and L. Legendre. 1998. Numerical Ecology. Elsevier, Amsterdam.

Lietti, M., J. C. Gamundi, G. Montero, A. Molinari, and V. Bulacio. 2008. Efecto de dos sistemas de labranza sobre la abundancia de artrópodos que habitan en el suelo. Ecología Austral 18:71-87.

Maceira, N. O., D. K. Zelaya, J. P. Celemín, and O. N. Fernández. 2005. Evaluación preliminar del uso de la tierra y elementos para el mejoramiento de la sustentabilidad: Reserva de la Biósfera de Mar Chiquita, Provincia de Buenos Aires. Informe final. Proyecto MAB/UNESCO.

Magura, T. 2002. Carabids and forest edge: spatial pattern and edge effect. Forest Ecology and Management 157:23- 
37.

Makarow, K. V. 1994. A key to the genera of the Ground-beetle larvae (Coleoptera, Carabidae) of the Palearctic region. Boll Mus Reg Sci Nat Torino 12:221-254.

Marasas, M. E. 2012. El camino hacia la transición agroecológica. Ediciones INTA, Ciudad Autónoma de Buenos Aires, Argentina.

Marasas, M. E., S. J. Sarandón, and A. C. Cicchino. 2001. Changes in soil functional groups in a wheat crop under conventional and no-tillage systems in Argentina. Applied Soil Ecology 18:61-68.

Marasas, M. E., S. J. Sarandón, and A. C. Cicchino. 2010. Semi-Natural Habitats and Field Margins in a Typical Agroecosystem of the Argentinean Pampas as a Reservoir of Carabid Beetles. Iournal of Sustainable Agriculture 34:1-16.

Montes, L., S. L. Alonso, M. C. Nuciari, A. M. Clausen, I. R. Guma, and A. M. Echarte. 2007. Flora espontánea del sudeste bonaerense. Universidad Nacional de Mar del Plata, Mar del Plata, Buenos Aires, Argentina.

Nanni, A. S. 2010. Variaciones espaciales y temporales de las comunidades de Carábidos en distintos tipos de uso de la tierra en el Bajo Delta del Río Paraná. Tesis de licenciatura. Facultad de Ciencias Exactas y Naturales, Universidad Nacional de Buenos Aires.

Niemelä, J. 2000. Biodiversity monitoring for decision-making. Annales Zoologici Fennici 37:307-317.

Niemelä, J. 2001. Carabid beetles (Coleoptera: Carabidae) and habitat fragmentation: a review. European Journal of Entomology 98:127-132.

Nisensohn, L., D. Faccini, E. Puricelli, D. Tuesca, L. Allieri, and S. Vecchi. 2009. Malezas de reciente difusión en los agroecosistemas de la región sojera núcleo. Parte I. Facultad de Ciencias agrarias, Universidad Nacional de Rosario. Dow AgroSciences, Buenos Aires, Argentina.

Paleologos, M. F. 2011. Los carábidos como componentes clave de la agrobiodiversidad. Su rol en la sustentabilidad de los agroecosistemas de vid de la zona de Berisso, Provincia de Buenos Aires. Tesis doctoral. Facultad de Ciencias Naturales y Museo, Universidad Nacional de La Plata, La Plata, Buenos Aires Argentina.

Pfiffner, L., and H. Luka. 2000. Overwintering of arthropods in soils of arable fields and adjacent semi-natural habitats. Agriculture, Ecosystems and Environment 78:215-222.

Porrini, D. P., A. V. Castro, and A. C. Cicchino. 2010. Distribución espacial y temporal de Paranortes cordicollis y Pachymorphus striatulus (Coleoptera: Carabidae) en dos ambientes edáficos de la Laguna Nahuel Rucá, Buenos Aires, Argentina. Actas XXII Congreso Argentino de la Ciencia del Suelo, Rosario, Santa Fe, Argentina.

Porrini, D. P., A. V. Castro, J. M. Arcusa, L. Peralta, G. Baloriani, and A. C. Cicchino. 2015. Aportaciones de los Agroecosistemas del Cinturón Hortícola Platense al Mantenimiento de la Diversidad y Funcionalidad de los Ensambles Locales de Carábidos (Insecta: Coleoptera). Actas del V Congreso Argentino de Agroecología, La Plata, Buenos Aires, Argentina.

Rainio, J., and J. Niemelä. 2003. Ground beetles (Coleoptera: Carabidae) as bioindicators. Biodiversity and Conservation 12:487-506.

Roig-Juñent, S., and J. Rouaux. 2012. A new species of Rhytidognathus (Carabidae, Migadopini) from Argentina. ZooKeys 247:45-60.

Sorensen, M. 2006. Composición y riqueza específica de carábidos (Coleoptera: Carabidae) en paisajes agrícolas de la E.E.A. INTA Balcarce, sudeste de Buenos Aires. Tesis de Licenciatura. Facultad de Ciencias Exactas y Naturales, Universidad Nacional de Mar del Plata, Mar del Plata, Buenos Aires, Argentina.

Stork, N. E., and P. Eggleton. 1992. Invertebrates as determinants and indicators of soil quality. American Journal of Alternative Agriculture 7:38-47.

Thiele, H. U. 1977. Carabid beetles in their environment. Zoophysiology and ecology 10. Springer Verlag, Berlin.

Thompson, R. G. 1979. Larvae of North American Carabidae with a Key to the Tribes. Pp. 209-291 en T.

Turienzo, P. N. 2006. Estudio de una comunidad estival de Carabidae (Insecta: Coleoptera) en seis ambientes diferentes del centro del partido de Balcarce (Buenos Aires). Tesis de Licenciatura. Facultad de Ciencias Agrarias, Universidad Nacional de Mar del Plata, Balcarce, Buenos Aires, Argentina.

Turin, H., K. Alders, P. J. Den Boer, S. Van Essen, T. H. Heijerman, W. Laane, and E. Penterman. 1991. Ecological characterisation of carabid species (Coleoptera, Carabidae) in the Netherlands from thirty years of pitfall sampling. Tijdschrift voor Entomologie 134:279-304.

Van Emden, F. I. 1942. A key to the genera pf larval Carabidae (Col.). Transactions of the Royal Entomological Society of London 92:1-99.

Venn, S. J. 2007. Morphological responses to disturbance in wing-polymorphic carabid species (Coleoptera: Carabidae) of managed urban grasslands. Baltic Journal of Coleopterology 7:51-59.

Venn, S. J., D. J. Kotze, and J. Niemelä. 2003. Urbanization effects on carabid diversity in boreal forests. European Journal of Entomology 100:73-80.

Wink, C., J. C. Guedes, C. K. Fagundes, and A. P. Rovedder. 2005. Insetos edáficos como indicadores da qualidade ambiental. Revista de Ciências Agroveterinárias 4:60-71. 